\title{
Exceptionally strong variation of galactic cosmic ray intensity at solar rotation period after the maximum of solar cycle 24
}

\author{
Agnieszka Gil ${ }^{* \dagger}$ \\ 1) Institute of Mathematics and Physics, Siedlce University, Poland \\ 2) ReSoLVE Centre of Excellence, Space Physics Research Unit, University of Oulu, Finland \\ E-mail: gila@uph.edu.pl
}

\section{Kalevi Mursula}

ReSoLVE Centre of Excellence, Space Physics Research Unit, University of Oulu, Finland

E-mail: kalevi.mursula@oulu.fi

\begin{abstract}
After the sunspot maximum and the reversal of solar polarity in 2014, the Sun is now in the early declining phase of cycle 24 . Soon after the polarity reversal, the galactic cosmic ray intensity, as observed, e.g., by neutron monitors at several latitudes (cut-off rigidities) depict an exceptionally large variation at the solar rotation period. This recurrence started in mid-2014 and continues until now (the first half of March 2015). Several parameters characterizing solar activity, like sunspots and F10.7 radio flux, also depict similar enhanced variability, which started slightly earlier than in neutron monitors. Some solar wind properties also show this periodicity, although less systematically and for a shorter time. This excessively strong periodicity in GCR can be related to the rapid growth of an asymmetric polar coronal hole in the southern hemisphere, leading to a very asymmetric magnetic configuration at mid- to high heliospheric latitudes. This also leads to the fact that the tilt angle of the heliospheric current sheet is more changeable, during this cycle than at similar early declining phases of the previous solar cycles.
\end{abstract}

The 34th International Cosmic Ray Conference,

30 July- 6 August, 2015

The Hague, The Netherlands

*Speaker.

${ }^{\dagger}$ A footnote may follow. 


\section{Introduction}

Galactic cosmic rays (GCR) measured on the ground by the worldwide network of neutron monitors (NMs) or by space-probes like AMS (e.g., [1]) or PAMELA (e.g., [2]) present variability in different time scales. As GCR stream is modulated by the Sun those variations coincide with solar cycles and other solar periodicities. Among them, there is GCR recurrence at solar rotation rate [3]. Although the rotation periodicity is often affected by other phenomena, there are sometimes periods when the solar rotation recurrence is very clear as during the solar cycle 23 in 2007-2008 (e.g., $[4,5])$. The strong variation of GCR intensity at solar rotation period was also very clearly seen after the maximum of solar cycle 24 , which is the topic of this paper.

\section{Data and analysis}

Solar rotation, the source of 27-day recurrence of GCR intensity, is not rigid, but differential, i.e. at various latitudes the rate of rotation is different. There are still many open questions about the solar differential rotation, such as its dependence on solar activity or the North-South asymmetry in differential rotation (e.g., [6],[7]). Investigation of recurrence of GCR intensity at solar rotation can help to shed some light on those issues.

To study the exceptionally strong variability of GCR intensity at solar rotation period we analyse daily NM count rates from the following stations at different latitudes and altitudes: Apatity (latitude $67.57^{\circ} \mathrm{N}$, altitude $181 \mathrm{~m}$ asl, with effective vertical cutoff rigidity $0.65 \mathrm{GV}$, from nmdb.eu), Jungfraujoch $\left(46.55^{\circ} \mathrm{N}, 3475 \mathrm{~m}\right.$ asl, $4.49 \mathrm{GV}$, from cosray.unibe.ch), Oulu $\left(65.05^{\circ} \mathrm{N}\right.$, $15 \mathrm{~m}$ asl, $0.8 \mathrm{GV}$, from cosmicrays.oulu.fi) and Rome $\left(41.86^{\circ} \mathrm{N}\right.$, sea level, $6.27 \mathrm{GV}$, from webusers.fis.uniroma3.it). Fig. 1a presents normalised daily count rates from Apatity NM (yellow curve with squares), Jungfraujoch (blue curve with crosses), Oulu (red curve with circles) and Rome (violet curve with vertical marks) in 01.01.2014-30.04.2015. Fig. 1b-1h display the main heliospheric and solar variability parameters (from omniweb.gsfc.nasa.gov), viz. strength of the heliospheric magnetic field (HMF) (B, Fig. 1b), components of HMF Bx, By, Bz (Fig. 1c-e, respectively), solar wind speed (Fig. 1f), sunspot number (Fig. 1g), solar radio flux (F 10.7-index, Fig. 1h) and, for comparison, the geomagnetic Kp index (Fig. 1i). Fig. 1 shows that recurrence at solar rotation period is prominent in GCR intensity and in all considered data during this early declining phase. In sunspot number (Fig. 1g) and solar radio flux (Fig. 1h) the solar rotation periodicity started to be clearly visible in April 2014 and lasted to January 2015, with a short break around November 2014. Cosmic rays (Fig. 1a) presented this variability a few months later, from August 2014 up to February 2015. The same behaviour as GCR is depicted by Bx (Fig. 1c) and By (Fig. 1d) components of HMF and solar wind speed (Fig. 1f). Sporadic Forbush decreases and other transients during that period (e.g., 10.09.2014 there was a strong solar flare associated with a coronal mass ejection on 13.09.2014 ) made the recurrence a bit disturbed, but still clearly visible. To show this periodicity even more evidently Fig. 2 displays the same data as Fig. 1, but detrended by subtracting a 41-days running average. To emphasise features of variability connected with solar rotation we performed a wavelet analysis (wavelet software was provided by Torrence and Compo [8], and is available at URL: paos.colorado.edu/research/wavelets/). Figs. 3-6 present examples of the wavelet analysis. Fig. 3 reveals strong solar rotation variability in Oulu NM daily data from 

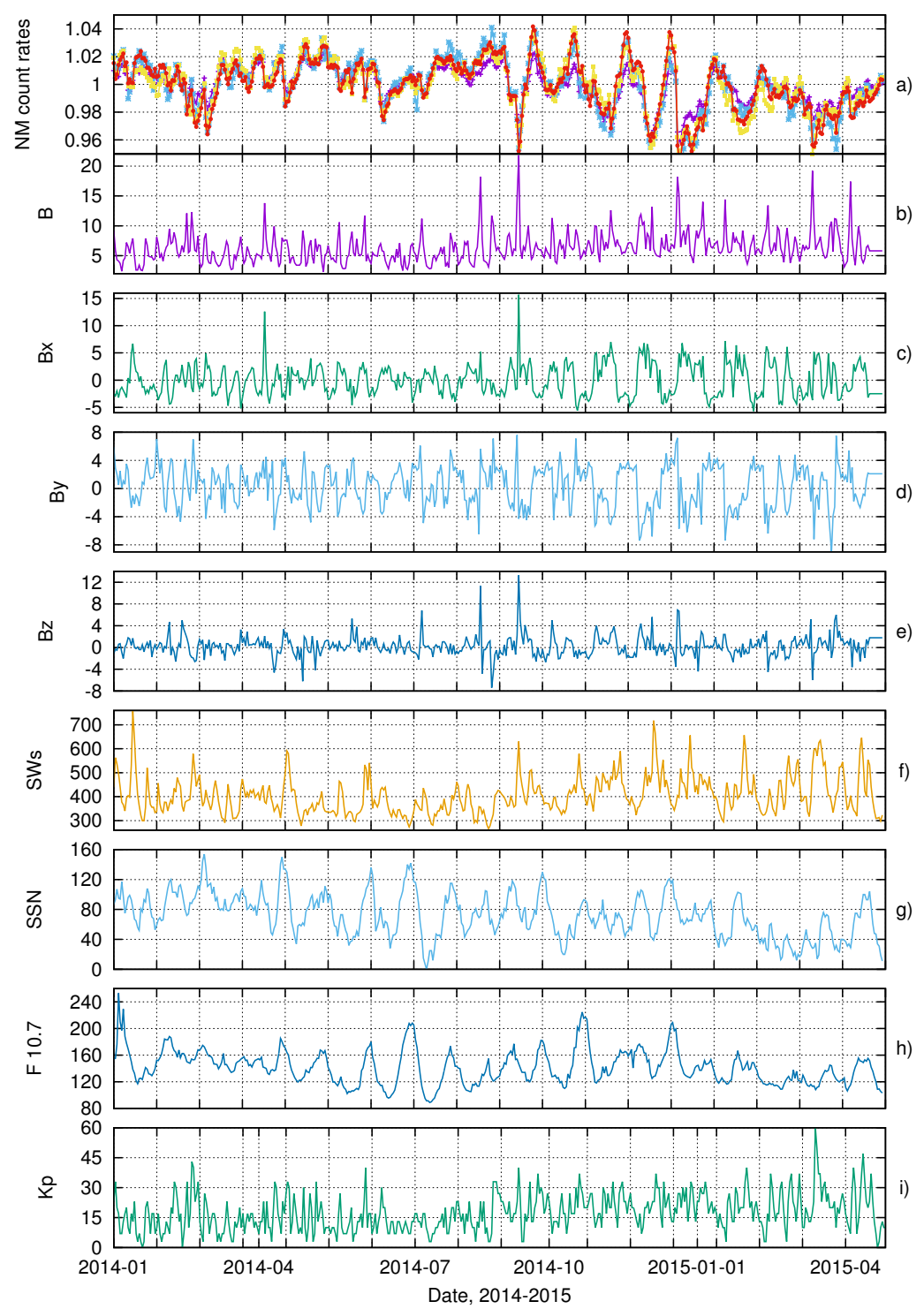

Figure 1: Daily data of a) cosmic rays NM count rates for different stations: Apatity (yellow curve with squares), Jungfraujoch (blue curve with crosses), Oulu (red curve with circles) and Rome (violet curve with vertical marks), b) strength of the heliospheric magnetic field B, c-e) components of HMF Bx, By, Bz, f) solar wind speed, g) sunspot number, h) solar radio flux (F 10.7) and i) geomagnetic Kp-index during period 01.01.2014-30.04.2015.

August 2014 up to February 2015. Fig. 5 shows that this recurrence appeared in solar wind speed slightly later, around October 2014, experiencing a shortening of duration. Fig. 6 confirms that recurrence at solar rotation period emerged in solar radio flux in April 2014 and was clearly visible till January 2015. Fig. 4 displays that the solar rotation recurrence was rather weakly visible in the HMF strength. To conform the wavelet analysis results we calculated power spectrum density (PSD) of all of the considered data using Lomb-Scargle algorithm [9, 10]. Figs. 7-10 present the PSDs for the same parameters as shown in Figs. 3-6 for a shorter interval 13.08.2014-03.03.2015, and Figs. 11-14 for the longer interval 01.01.2014-30.04.2015. The most significant periodicities for all of the considered data are gathered in Table 1. Tab. 1 and Fig. 7 show that the main period- 


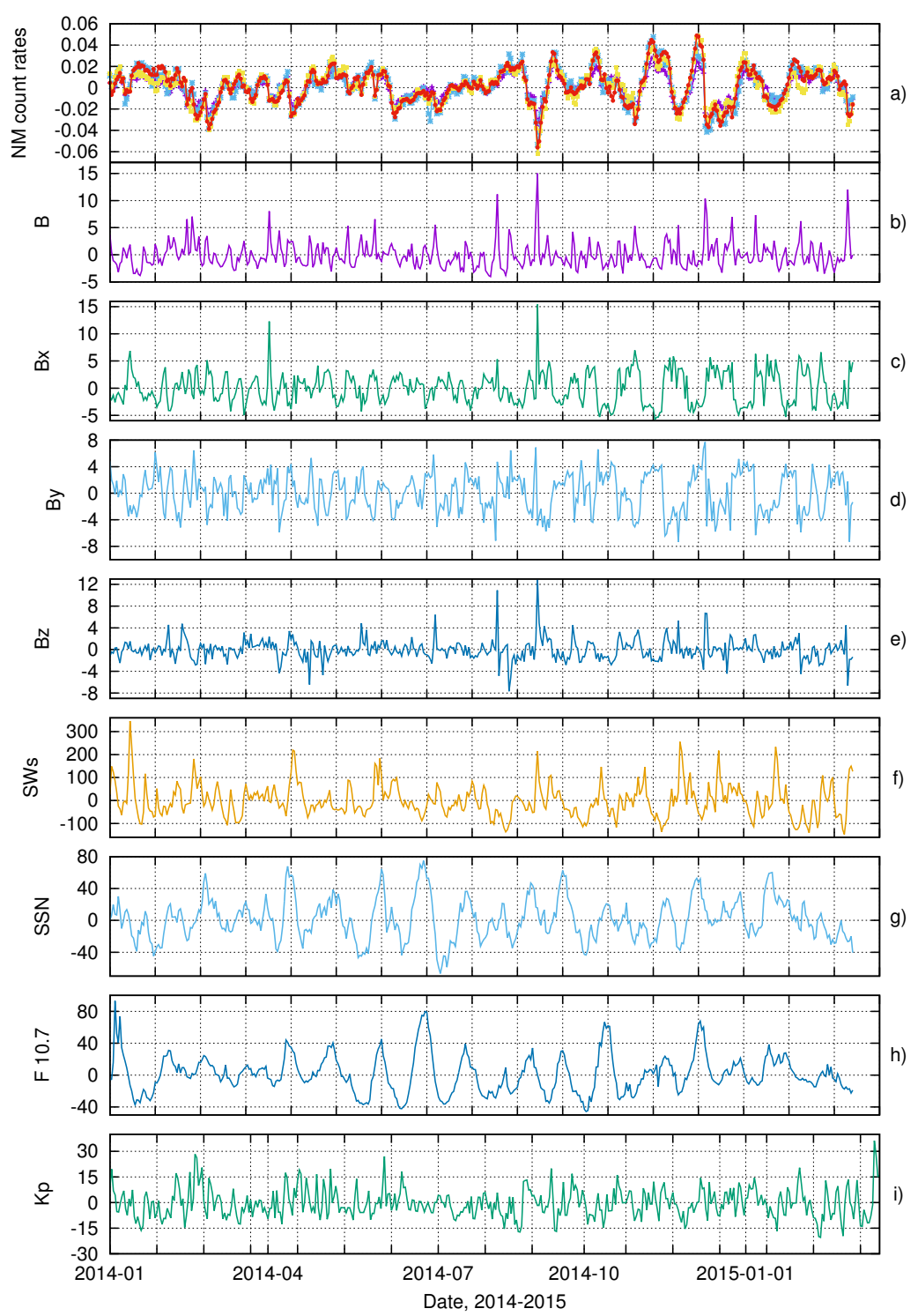

Figure 2: Detrended with 41- days moving average daily data of a) cosmic rays NM count rates for different stations: Apatity (yellow curve with squares), Jungfraujoch (blue curve with crosses), Oulu (red curve with circles) and Rome (violet curve with vertical marks), b) strength of the heliospheric magnetic field B, c-e) components of HMF Bx, By, Bz, f) solar wind speed, g) sunspot number, h) solar radio flux (F 10.7) and i) geomagnetic Kp-index during period 01.01.2014-30.04.2015.

icity in GCR intensity during 13.08.2014-03.03.2015 was 28.857 days. The periodicities in other parameters during this period are shorter, especially for the HMF strength. For the longer period, 01.01.2014-30.04.2015, Figs. 11-14 and Tab. 1 display that even a main periodicity of cosmic rays is longer than in all other heliospheric and solar variability parameters, except for the HMF strength.

\section{Polar coronal holes and GCR recurrence at solar rotation period.}

It is well known that a recurrence at solar rotation period in GCR intensity is related to coro- 


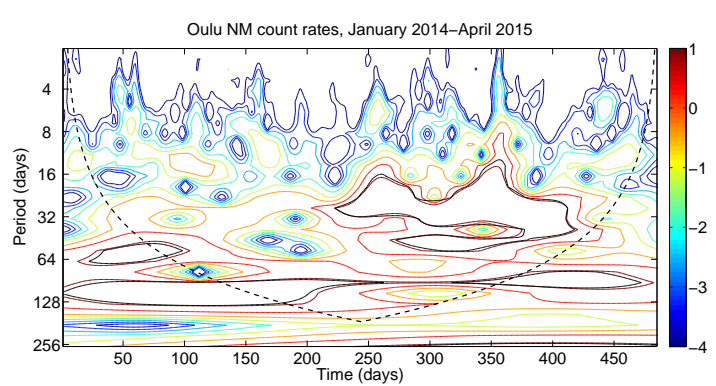

Figure 3: Wavelet analysis for Oulu NM daily data during period 01.01.2014-30.04.2015.

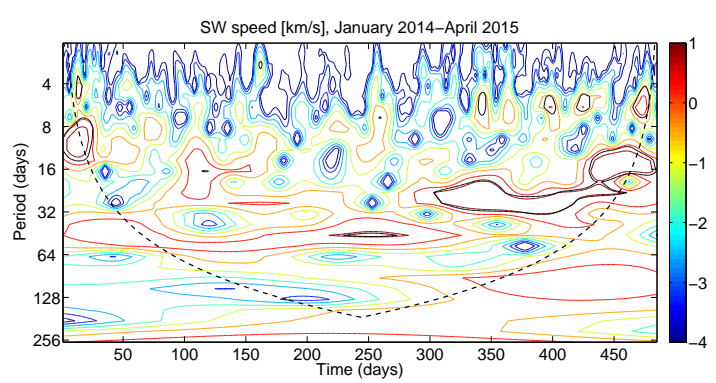

Figure 5: Wavelet analysis for solar wind speed daily data during period 01.01.2014-30.04.2015.

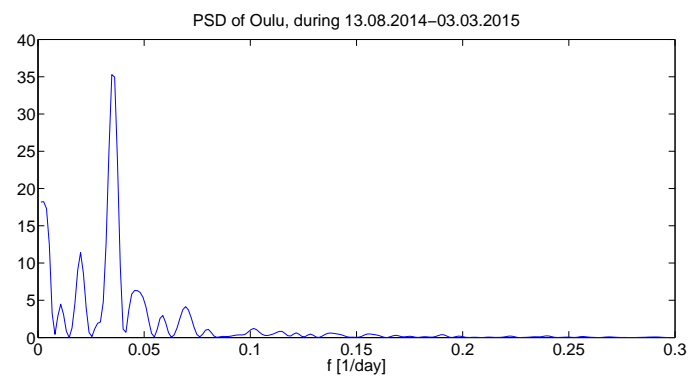

Figure 7: PSD of Oulu NM daily data during period 13.08.2014-03.03.2015.

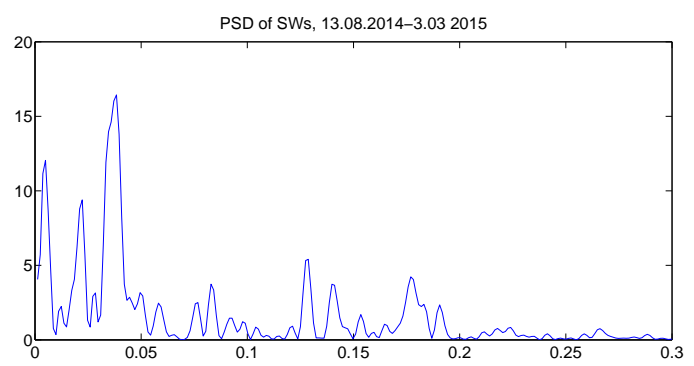

Figure 9: PSD of solar wind speed daily data during period 13.08.2014-03.03.2015.

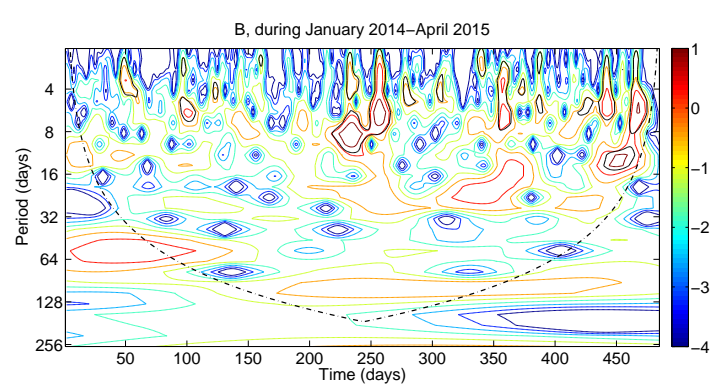

Figure 4: Wavelet analysis for the HMF strength, B, daily data during period 01.01.2014-30.04.2015.

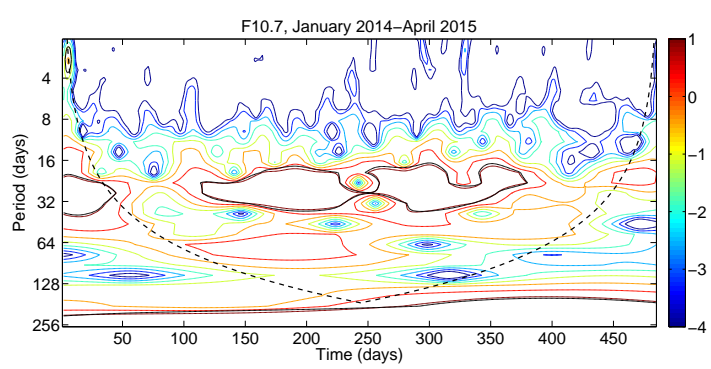

Figure 6: Wavelet analysis for solar radio flux (F 10.7) daily data during period 01.01.2014-30.04.2015.

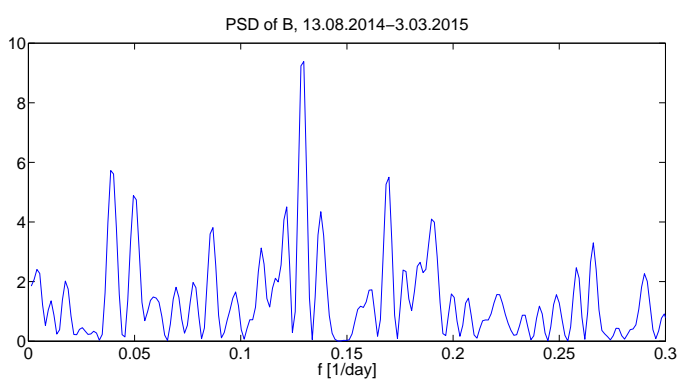

Figure 8: PSD of the HMF strength, B daily data during period 13.08.2014-03.03.2015.

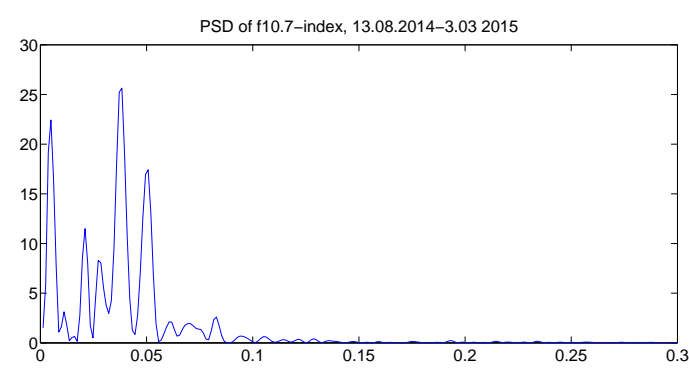

Figure 10: PSD of solar radio flux daily data during period 13.08.2014-03.03.2015. 
nal holes and their development in time (e.g., [11]). They modify the structure of the high-speed solar wind streams and modulate GCR stream. During periods when there exists one predominant coronal hole producing high-speed solar wind near the ecliptic, the first harmonic in recurrence of GCR intensity is likely to enhance.

We analysed the EUV full-disk images obtained by SoHO EIT (from umbra.nascom.nasa.gov) for a few days before each minimum in the cosmic rays during the period of 13.08.2014-03.03.2015. We found that every time before a decrease in cosmic rays appeared, three-four days earlier there was a large coronal hole visible in the southern hemisphere. Thus, a clear North-South asymmetry in polar coronal hole occurrence leading to a very asymmetric magnetic configuration at mid- to high heliospheric latitudes is a very probable source of this strong periodicity in GCR. Moreover, it is worth mentioning that polar field reversal process was also asymmetric. Sun et al. [12] described that the reversal of northern polar fields was in November 2012 and southern, about sixteen months later, in March 2014. This asymmetric magnetic configuration at mid- to high heliospheric latitudes also led to the fact that the tilt angle of the heliospheric current sheet, as shown in Fig. 15 (from wso.stanford.edu), was more variable during this early declining phase compared to the three previous solar cycles.

\section{Summary}

During the early declining phase of solar cycle 24 GCR intensity measured by neutron monitors with different cut-off rigidities presented a very strong variability at solar rotation period. This periodicity started in August 2014 and continued to the beginning of March 2015. Parameters indicating solar activity, as sunspot number and solar radio flux, also displayed similar recurrence, which started earlier than in neutron monitors, in April 2014. Moreover, Bx and By components of HMF, as well as, solar wind speed also showed this variability. We explain this enhanced solar rotation periodicity in GCR with the fairly rapid growth of an asymmetric southern polar coronal hole, which led to an asymmetric magnetic configuration at mid- to high heliolatitudes. We also note that the tilt angle of the HCS was more variable in this cycle than during the similar epoch of the recent solar cycles.

\section{Acknowledgments}

Data of neutron monitor count rates are from Oulu NM, http://cosmicrays.oulu.fi, Jungfraujoch from http://cosray.unibe.ch, Rome from http://www.webusers.fis.uniroma3.it and Apatity from http://www.nmdb.eu. Measurements of heliospheric and solar variability parameters are from http://omniweb.gsfc.nasa.gov, tilt angle of HCS from http://wso.stanford.edu and SoHO EUV images from http://umbra.nascom.nasa.gov. We acknowledge the financial support by the Academy of Finland to the ReSoLVE Centre of Excellence (project no. 272157). This work was partially supported by the Foundation for Polish Science. 


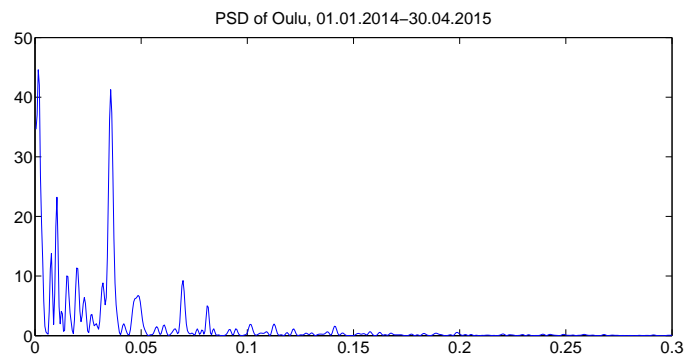

Figure 11: PSD of Oulu NM daily data during period 01.01.2014-30.04.2015.

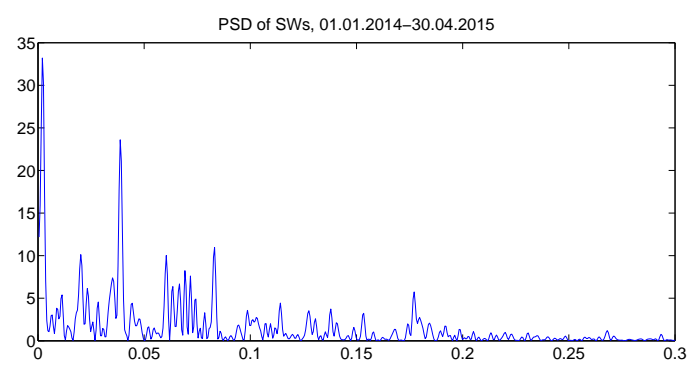

Figure 13: PSD of solar wind speed daily data during period 01.01.2014-30.04.2015.

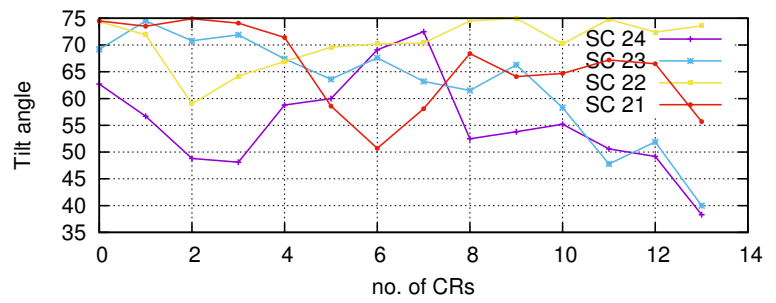

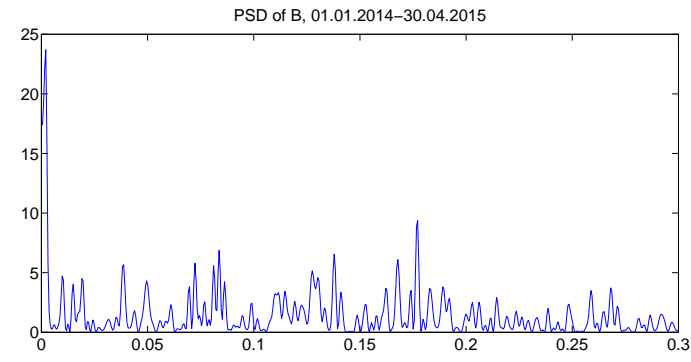

Figure 12: $\mathrm{PSD}$ of the HMF strength, B, daily data during period 01.01.2014-30.04.2015.

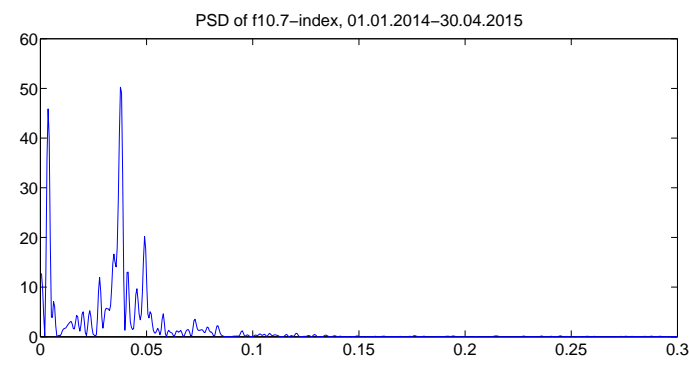

Figure 14: PSD of solar radio flux daily data during period 01.01.2014-30.04.2015.

Figure 15: Changes of the heliospheric current sheet tilt angle in early declining phases during the recent four solar cycles. For each SC a 0 rotation on the ordinate means a rotation during which the sunspots maximum occurred, namely Carrington rotation no. 2149 in SC 24, CR 1961 in SC 23, CR 1818 in SC 22 and CR 1690 in SC 21.

\section{References}

[1] Aguilar, M., Aisa, D., Alpat, B., and 294 coauthors, Precision Measurement of the Proton Flux in Primary Cosmic Rays from Rigidity $1 \mathrm{GV}$ to $1.8 \mathrm{TV}$ with the Alpha Magnetic Spectrometer on the International Space Station, PhRvL 114 171103, 2015

[2] Picozza, P., A. M. Galper, G. Castellini, and 57 coauthors, PAMELA-A payload for antimatter matter exploration and light-nuclei astrophysics, Astropart. Phys. 27 (4) 296, 2007

[3] Forbush, S. E., On cosmic-ray effects associated with magnetic storms, TeMAE 43 203, 1938

[4] Leske, R., Cummings, A. C., Mewaldt, R. A., Stone, E. C., von Rosenvinge, T. T., \& Wiedenbeck, M. E., Observations of 27-Day Variations in Cosmic Ray Intensities During the Cycle 23/24 Solar Minimum, Proceedings of the 32nd ICRC2011 11 194, 2011

[5] Modzelewska, R. \& Alania, M. V., The 27-Day Cosmic Ray Intensity Variations During Solar Minimum 23/24 Solar Phys. 286 593, 2013 
Table 1: Comparison of the most significant periodicities (in days) for all of the considered data during the periods of 13.08.2014-03.03.2015 and 01.01.2014-30.04.2015

\begin{tabular}{lll}
\hline Data & Main periodicity, & Main periodicity, \\
set & $\mathbf{0 1 . 2 0 1 4 - 0 4 . 2 0 1 5}$ & $\mathbf{1 3 . 0 8 . 2 0 1 4 - 0 3 . 0 3 . 2 0 1 5}$ \\
\hline Oulu NM & 28.057 & 28.857 \\
Apatity NM & 28.057 & 28.857 \\
Rome NM & 28.057 & 28.857 \\
Jungfraujoch NM & 28.057 & 28.857 \\
solar wind speed & 25.813 & 26.064 \\
solar wind proton density & 26.162 & 26.064 \\
solar wind proton temperature & 25.813 & 26.064 \\
HMF strength, B & 52.324 & 7.769 \\
HMF component, Bx & 26.889 & 26.933 \\
HMF component, By & 26.889 & 26.933 \\
HMF component, Bz & 24.820 & 25.253 \\
sunspot number & 26.162 & 20.200 \\
solar radio flux (F 10.7 index) & 26.520 & 26.064 \\
Kp-index & 25.813 & 25.250 \\
\hline
\end{tabular}

[6] Suzuki, M., On the Long-Term Modulation of Solar Differential Rotation, Solar Phys. 289 4021, 2014

[7] Zhang, L., K. Mursula, \& I. Usoskin, Consistent long-term variation in the hemispheric asymmetry of solar rotation, $A \& A \mathbf{5 5 2}$ id.A84, 2013

[8] Torrence, C., \& Compo, G. P., A Practical Guide to Wavelet Analysis, Am. Meteorol. Soc. 79 61, 1998

[9] Lomb, N. R., Least-squares frequency analysis of unequally spaced data, Ap\&SS 39 447, 1976

[10] Scargle, J. D., Studies in astronomical time series analysis. I - Modeling random processes in the time domain, Ap. J. 45 1, 1981

[11] Kunow, H., Dröge, W., Heber, B., et al., High energy cosmic-ray nuclei results on Ulysses: 2. Effects of a recurrent high-speed stream from the southern polar coronal hole, Space Sci. Rev. 72 397, 1995

[12] Sun, X., Hoeksema, J. T., Liu, Y., \& Zhao, J., On Polar Magnetic Field Reversal and Surface Flux Transport During Solar Cycle 24, Ap. J. 798 114, 2015 